\title{
Role of Versican and ADAMTS-1 in Polycystic Ovary Syndrome
}

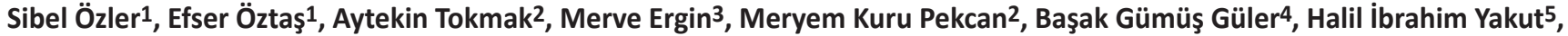 \\ Nafiye Yılmaz 2 \\ 1Zekai Tahir Burak Women's Health Training and Research Hospital, Clinic of Perinatology, Ankara, Turkey \\ 2Zekai Tahir Burak Women's Health Training and Research Hospital, Clinic of Obstetrics and Gynecology, Ankara, Turkey \\ 325 Aralık State Hospital, Clinic of Clinical Biochemistry, Gaziantep, Turkey \\ 4 Liv Hospital, Clinic of Obstetrics and Gynecology, Ankara, Turkey \\ 5Zekai Tahir Burak Women's Health Training and Research Hospital, Clinic of Pediatrics, Ankara, Turkey
}

\section{What is already known on this topic?}

Patients having polycystic ovary syndrome (PCOS), show the symptoms of oligo/anovulation, and hyperandrogenism; and their ultrasonographic examination demonstrates the polycystic view of ovaries. The degradation of cumulus oophorus complex (COC) during ovulation, depends on excretion of various cytokines, prostoglandins, extracellular matrix enzymes, and proteases from the neighboring cells. Versican is one of the proteoglycans forming the COC; and it is degraded by the protease, ADAMTS-1, during the ovulation process.

\section{What this study adds?}

Our aim in this study was to investigate if serum versican and ADAMTS-1 levels could be used as a serum marker in anovulation patients. We consider these markers to be used in the treatment strategies of infertile patients having PCOS; with the help of upcoming studies, containing more sample sizes.

\section{Abstract}

Objective: ADAMTS-1 is a matrix metalloproteinase which cleaves versican in the cumulus oocyte complex under the effect of luteinizing hormone surge in the periovulatory period. Altered levels may have a role in the pathogenesis of polycystic ovary syndrome (PCOS). We aimed to determine the serum versican and ADAMTS-1 (a disintegrin and metalloproteinase with thrombospondin motif-1) levels in PCOS patients and compare the results with healthy controls.

Methods: Thirty-eight patients with PCOS and forty healthy controls aged between 15 and 22 years were included in the study. They were sampled according to their basal hormone, serum versican, and ADAMTS-1 levels. Serum versican and ADAMTS- 1 levels were measured by enzyme-linked immunosorbent assay. A multivariate logistic regression model was used to identify the independent risk factors of PCOS.

Results: Serum versican levels were significantly decreased in the PCOS group when compared with the controls. The best versican cut-off value for PCOS was calculated to be 33.65 with $76.74 \%$ sensitivity and $52.94 \%$ specificity. Serum versican levels, homeostasis model assessment of insulin resistance index, a Ferriman-Gallwey score higher than 8 , and oligomenorrhea were the strongest predictors of PCOS. Serum versican levels were significantly decreased in PCOS patients. Besides, serum ADAMTS-1 and versican levels were significantly and positively correlated with each other.

Conclusion: Serum versican levels were significantly decreased in patients with PCOS. This suggests a possible role of versican in ovulatory dysfunction and in the pathogenesis of PCOS.

Keywords: Versican, ADAMTS-1, polycystic ovary syndrome 


\section{Introduction}

Polycystic ovary syndrome (PCOS) is one of the most frequent endocrinopathies among women of reproductive age, with a prevalence of $6-26 \%$, depending on the diagnostic criteria and ethnicity $(1,2)$. Women with PCOS have increased risk of developing type 2 diabetes. PCOS is also associated with multiple cardiovascular risk factors $(3,4)$. In many women with PCOS, the signs and symptoms start at adolescence (5). Considering the adverse long-term consequences of PCOS, especially when the symptoms begin in the early period of life, early diagnosis and treatment are of great importance for prevention of its progression. It is important to know the underlying factors causing PCOS to provide guidance for early intervention. Although genetic and environmental factors are thought to have a role in the etiopathogenesis of the disease, the exact mechanism is still unclear (5).

PCOS is defined as a syndrome of ovarian dysfunction and its clinical manifestations are mainly attributed to this (4). During both fetal and adult life, continuous remodeling of the ovarian tissue is taking place to maintain normal ovarian function, and this process requires changes also in the extracellular matrix (ECM) (6). Successful ovulation is a complex process consisting of follicular development, ovulation, luteal formation and subsequent regression, and these are all dependent on the cyclical remodeling of ECM (7). Most critical ovulatory mediators were shown to be the ones exerting their effects through the cumulus cell complex surrounding the oocyte (8). Besides, it has been suggested that extensive tissue remodeling of the ovary is mediated by heparan sulfate proteoglycans and matrix metalloproteinases (MMP) $(7,9,10)$.

A disintegrin-like metalloproteinase with thrombospondin motif (ADAMTS) proteases are a distinct group of zinc metalloproteases, comprising 20 members and known to function in the cleavage and degradation of various ECM components (11). Increasing evidence suggests that ADAMTSs have a role in various physiopathological processes such as morphogenesis during embryonic development, follicular development and ovulation, cyclic endometrial remodeling, angiogenesis as well as in development of cancer, of thrombotic and inflammatory conditions $(11,12,13,14,15)$. ADAMTS- 1 is a MMP that has been implicated in the inhibition of angiogenesis induced by luteinizing hormone $(\mathrm{LH})$ in the periovulatory follicles of the mouse and rat and is also thought to be a mediator of proteolytic cleavage of the hyaluronan binding proteoglycans, aggrecan, and versican $(16,17)$.

Cumulus oocyte complex (COC) is rich of hyaluronan which is synthesized by cumulus cells, via expression of hyaluronan synthase-2 (18). Versican, a large hyaluronic acid (HA) binding proteoglycan, is expressed by periovulatory granulosa cells and localized within the expanding matrix of COC (18). The secreted active form of ADAMTS- 1 has been shown to be localized selectively in COC, and with the effect of LH surge in the periovulatory period, to lead to cleavage of versican (19). Thus, it has been suggested that cleavage of versican in the expanded $\mathrm{COC}$ matrix is an important function of ADAMTS-1 in the ovulation process $(18,19)$. Furthermore, previous studies suggested a relationship between versican and atherosclerosis, diabetes, insulin resistance (IR) and endothelial dysfunction, which are wellknown long-term consequences of PCOS $(3,20,21)$.

Based on the results of the above-mentioned studies, we hypothesized that altered levels of ADAMTS-1 and versican in the ECM of $\mathrm{COC}$, by causing ovulatory dysfunction, might be causative factors for the clinical manifestations of PCOS. In the present study, we aimed to compare the serum ADAMTS-1 and versican levels in adolescent and young females with PCOS with those of a control group.

\section{Methods}

Thirty-eight adolescent and young female subjects with PCOS, aged between 15-22 years, were recruited consecutively from the outpatient clinic of Zekai Tahir Burak Women's Health Training and Research Hospital. The diagnosis of PCOS was based on presence of two of the following three criteria: oligo- or anovulation, clinical and/ or biochemical signs of hyperandrogenism, and polycystic ovaries, as proposed by the Rotterdam consensus in 2003 (4). Venous blood from the patients was sampled on the third day of their menstrual cycles. Forty age-matched healthy controls were also recruited.

Exclusion criteria were use of medications known to alter insulin secretion or action and lipoprotein metabolism; hypertension; smoking; family history of cardiovascular diseases; and endocrinopathies including diabetes, Cushing syndrome, androgen-secreting tumors, late-onset 21-hydroxylase deficiency, thyroid dysfunction; current use of oral contraceptives; presence of autoimmune diseases; and hyperprolactinemia. All participants provided a written informed consent and the study protocol was approved by the local ethics committee of our hospital (Approval date/ number: 16.10.2014/15).

Clinical examinations were performed and anthropometric measurements were recorded for all participants included in the study. Body mass index (BMI) was calculated by using the formula: weight $(\mathrm{kg}) /$ height $\left(\mathrm{m}^{2}\right)$. Waist circumference 
(WC) was measured as the circumference of the abdomen at its narrowest point between the lower costal $\left(10^{\text {th }}\right.$ rib) border and the top of the iliac crest. Hip circumference was measured at the level of greatest posterior protuberance of the buttocks. Blood samples were obtained by venipuncture after an overnight fasting for at least 12 hours for biochemical evaluation and were processed within 1 hour after withdrawal. The serum samples were stored at $-80{ }^{\circ} \mathrm{C}$ until the day of analysis.

All analyses were performed with the use of a Beckman Coulter (High Wycombe, United Kingdom) Gen-S automated analyzer. Plasma glucose levels were determined with the glucose hexokinase method. Serum levels of folliclestimulating hormone (FSH), and $\mathrm{LH}$ were determined by immunochemiluminometric assay. Inter-/intra-assay coefficients of variability (CV) for FSH and LH were $2.3 \% / 1.4 \%$ and $2.1 \% / 3.1 \%$, respectively. Estradiol, prolactin, dehydroepiandrosterone sulfate (DHEAS), total testosterone (total-T), insulin, and thyroid-stimulating hormone (TSH) were measured using the UniCel DxI 800 radioimmunoassay system (Beckman Coulter, Fullerton, CA, USA). The inter- and intra-assay CVs were $0.1 \%$ and $3.2 \%$ for estradiol, $1.7 \%$ and $3.2 \%$ for prolactin, $1.7 \%$ and $2.8 \%$ for DHEAS, $0.5 \%$ and $1.7 \%$ for total-T, $8.5 \%$ and $2.4 \%$ for TSH. Serum levels of 17-hydroxy progesterone $(17 \mathrm{OH}-\mathrm{P})$ and free testosterone (free-T) were measured by radioimmunoassay (Siemens, Erlangen, Germany). The inter- and intra-assay CVs were $4.6 \%$ and $10.7 \%$ for $17 \mathrm{OH}$ $\mathrm{P}$ and $5.7 \%$ and $11.4 \%$ for free-T, respectively. Homeostasis model assessment of IR (HOMA-IR) (insulin $\times$ glycemia in $(\mu \mathrm{mol} / \mathrm{L}) / 22.5)$ was estimated. HOMA-IR $>2.5$ was considered to indicate the presence of IR (22). The serum levels of total cholesterol, high-density lipoprotein (HDL), low-density lipoprotein (LDL), and triglycerides (TG) were determined with enzymatic colorimetric assays via the use of an AU680 Chemistry System (Beckman Coulter, Fullerton, CA, USA). The lipid accumulation product (LAP) index was calculated using the formula [WC $(\mathrm{cm})-58] \times[\mathrm{TG}$ concentration (mmol/L)] (23).

Serum ADAMTS- 1 concentrations were determined by human ADAMTS-1 enzyme-linked immunosorbent assay (ELISA) (Eastbiopharm Co., Ltd., Hangzhou, China) and the results were expressed as $n g / m L$. Serum versican concentrations were determined by using human versican ELISA kit (Boster Biological Technology Co., California, USA) and the results were expressed as $\mathrm{pg} / \mathrm{mL}$.

\section{Statistical Analysis}

Data analysis was performed by using Statistical Package for the Social Sciences for Windows, version 11.5 (SPSS
Inc., Chicago, IL, United States). The data were shown as mean [95\% confidence interval (CI)] or number of cases and (percentage), where applicable. The KolmogorovSmirnov test was used to determine whether continuous variables were normally distributed or not. Homogeneity of variances was evaluated by the Levene test. Continuous variables were shown as mean \pm standard deviation (SD) or median (minimum-maximum), where applicable. Mean differences between case and control groups were compared by student's t-test. Mann-Whitney U-test was used to compare the median values. Nominal data were analyzed by Pearson's chi-square test. Whether the mean differences between groups were statistically significant or not, were evaluated by analysis of covariance (ANCOVA). Degrees of association between continuous variables were evaluated by partial correlation analyses. The optimal cutoff points of laboratory parameters discriminating groups were evaluated by receiver operating characteristic (ROC) analyses, calculating area under the curve (AUC) as giving the maximum sum of sensitivity and specificity for the significant test. Multiple logistic regression analyses were applied for calculating odds ratios (OR) and 95\% CIs for each clinical condition. Linear regression model was used to evaluate the relation of independent variables as clinical and laboratory parameters in the group having PCOS. A p-value less than 0.05 was considered statistically significant.

\section{Results}

A total of seventy-eight participants (38 adolescent and young females with PCOS and 40 age-matched healthy controls) were enrolled in this case-control study. The baseline clinical, endocrinological, and laboratory characteristics are given in Table 1.

Among the laboratory parameters, fasting plasma glucose, insulin, HOMA-IR, estradiol, and LH levels were significantly higher in PCOS group. LAP index (calculated as 29.86 and 14.17 in PCOS and control groups, respectively) was also significantly higher in PCOS patients $(p<0.001)$. The mean value for $W C$ in PCOS group was $77.23 \pm 11.87 \mathrm{~cm}$, which was significantly higher than that of the control group, $72.89 \pm 7.78 \mathrm{~cm}(p=0.041)$. ADAMTS-1 levels were not significantly lower in PCOS group $(p=0.959)$. The serum versican levels of the PCOS and control groups were detected as $54.69 \mathrm{ng} / \mathrm{mL}$ and $95.6 \mathrm{ng} / \mathrm{mL}$, respectively, showing that serum versican levels were significantly decreased in PCOS group when compared with the controls $(p=0.009)$.

Versican levels were re-evaluated using ROC analysis; cut-off levels were determined and AUC was calculated. According to 
Table 1. Baseline characteristics, clinical and laboratory parameters of patients with polycystic ovary syndrome and controls

\begin{tabular}{|c|c|c|c|}
\hline & $\operatorname{PCOS}(n=38)$ & Control $(n=40)$ & $\mathrm{p}$-value \\
\hline \multicolumn{4}{|l|}{ Clinical findings } \\
\hline Age (years) & $18.38 \pm 2.07$ & $18.89 \pm 2.59$ & 0.320 \\
\hline Age at menarche (years) & $12.67 \pm 1.10$ & $13.02 \pm 1.17$ & 0.160 \\
\hline BMI $\left(\mathrm{kg} / \mathrm{m}^{2}\right)$ & $23.52 \pm 2.91$ & $22.95 \pm 3.70$ & 0.439 \\
\hline WHR & $0.79 \pm 0.07$ & $0.76 \pm 0.06$ & 0.068 \\
\hline WC (cm) & $77.23 \pm 11.87$ & $72.89 \pm 7.78$ & 0.041 \\
\hline \multicolumn{4}{|l|}{ Laboratory findings } \\
\hline Fasting blood glucose (mg/dL) & $88.5(76-105)$ & $86(64-127)$ & 0.034 \\
\hline Insulin $(\mu \mathrm{IU} / \mathrm{mL})$ & $10.14(4.72-46.7)$ & $7.05(2.43-18.79)$ & 0.004 \\
\hline HOMA-IR & $2.13(0.98-9.69)$ & $1.63(0.49-3.94)$ & 0.004 \\
\hline Estradiol (pg/mL) & $36.82 \pm 17.90$ & $25.73 \pm 17.38$ & 0.008 \\
\hline LH (mIU/mL) & $8.19(3.56-21.13)$ & $4.66(0.65-31.77)$ & $<0.001$ \\
\hline FSH (mIU/mL) & $6.25(2.32-12.60)$ & $6.27(2.74-21.18)$ & 0.897 \\
\hline DHEA-S $(\mu \mathrm{g} / \mathrm{dL})$ & $386(137.1-953.7)$ & $313.7(179.7-466.3)$ & 0.075 \\
\hline 17-OH progesterone $(\mathrm{ng} / \mathrm{dL})$ & $1.23(0.71-8.01)$ & $0.92(0.27-2.83)$ & 0.073 \\
\hline Total-C (mg/dL) & $156.03 \pm 27.06$ & $151.92 \pm 31.08$ & 0.555 \\
\hline LDL-C (mg/dL) & $72.70 \pm 23.54$ & $70.32 \pm 25.33$ & 0.677 \\
\hline HDL-C (mg/dL) & $63.80 \pm 8.23$ & $64.63 \pm 9.41$ & 0.728 \\
\hline TG-C (mg/dL) & $85(45-298)$ & $71.5(39-284)$ & 0.081 \\
\hline Total cholesterol/HDL-C ratio & $2.31(1.68-3.70)$ & $2.2(1.68-4.82)$ & 0.357 \\
\hline LAP index & $29.86(1.66-191.94)$ & $14.17(-2.28-65.45)$ & $<0.001$ \\
\hline Total testosterone & $18.78 \pm 11.52$ & $20.61 \pm 9.98$ & 0.549 \\
\hline Free testosterone & $1.46(0.83-5.77)$ & $1.36(0.60-3.57)$ & 0.075 \\
\hline ADAMTS-1 (pg/mL) & $4.63(0.14-15.8)$ & $5.03(1.63-17.02)$ & 0.959 \\
\hline Versican (ng/mL) & $54.69(21.56-158.9)$ & $95.6(25.1-283.0)$ & 0.009 \\
\hline
\end{tabular}

*Student's t-test; mean \pm standard deviation. Mann-Whitney U-test; median (minimum-maximum). p-value < 0.05 is considered as statistically significant; BMI: body mass index, WC: waist circumference, WHR: waist to hip ratio, HOMA-IR: homeostasis model assessment of insulin resistance, LH: luteinizing hormone, FSH: follicle-stimulating hormone, TSH: thyroid-stimulating hormone, DHEA-S: dehydroepiandrosterone sulfate, Total-C: total cholesterol, LDL-C: low-density lipoprotein cholesterol, HDL-C: high-density lipoprotein cholesterol, TG-C: triglycerides-cholesterol, LAP index: lipid accumulation product index, ADAMTS-1: a disintegrin and metalloproteinase with thrombospondin motif-1, 17-OH: 17-hydroxy

Table 2. Regression analysis in the prediction of polycystic ovary syndrome

\begin{tabular}{lllll}
\hline & Univariate & & Multivariate \\
\cline { 2 - 5 } & OR $\mathbf{( 9 5 \% ~ C I )}$ & p-value & OR $\mathbf{( 9 5 \% ~ C I ) ~}$ & p-value \\
\hline HOMA-IR $>2.5$ & $0.212(0.053-0.852)$ & $\mathbf{0 . 0 2 9}$ & $1.452(0.476-4.429)$ & 0.512 \\
Estradiol $(\mathrm{pg} / \mathrm{mL})$ & $1.037(1.008-1.067)$ & $\mathbf{0 . 0 1 1}$ & $1.034(0.987-1.084)$ & 0.158 \\
LH $(\mathrm{mIU} / \mathrm{mL})$ & $1.153(1.026-1.296)$ & $\mathbf{0 . 0 1 7}$ & $1.248(1.029-1.513)$ & $\mathbf{0 . 0 2 4}$ \\
LAP index & $1.062(1.019-1.107)$ & $\mathbf{0 . 0 0 4}$ & $1.038(0.982-1.098)$ & 0.187 \\
Versican $(\mathrm{ng} / \mathrm{mL})$ & $0.990(0.981-0.998)$ & $\mathbf{0 . 0 1 8}$ & $0.971(0.949-0.994)$ & $\mathbf{0 . 0 1 5}$ \\
\hline
\end{tabular}

p-value < 0.05 is considered as statistically significant; HOMA-IR: homeostasis model assessment of insulin resistance, LH: luteinizing hormone, LAP index: lipid accumulation product index, OR: odds ratio, CI: confidence interval

the ROC analysis performed for the diagnostic performance of serum versican levels for PCOS, the AUC was $0.675(95 \%$ CI: $0.55-0.795 ; p=0.009$ ) (Figure 1 ). The best versican cut-off value for PCOS was 33.65 with $76.74 \%$ sensitivity,
$52.94 \%$ specificity, $97.35 \%$ positive and $64.29 \%$ negative predictive values. According to the ROC analysis performed to assess the differences of LAP index between groups, the AUC was 0.714 (95\% CI: 0.601-0.827; p<0.001) (Figure 2). 
Table 3. Linear regression model for the assessment of polycystic ovary syndrome risk

\begin{tabular}{llllll}
\hline & \multicolumn{5}{c}{ Unstandardized coefficients } \\
\cline { 2 - 6 } & $\beta$ & $\begin{array}{l}\text { Standard } \\
\text { error }\end{array}$ & Beta & t & p-value \\
\hline Versican & -0.002 & 0.001 & -0.333 & -3.348 & $\mathbf{0 . 0 0 1}$ \\
HOMA-IR & 0.085 & 0.029 & 0.293 & 2.984 & $\mathbf{0 . 0 0 4}$ \\
FGS & 0.034 & 0.010 & 0.341 & 3.438 & $\mathbf{0 . 0 0 1}$ \\
Oligomenorrhea & 0.305 & 0.107 & 0.286 & 2.846 & $\mathbf{0 . 0 0 6}$ \\
\hline HOMA-IR: homeostasis model assessment of insulin resistance, \\
FGS: Ferriman Galloway score \\
\hline
\end{tabular}

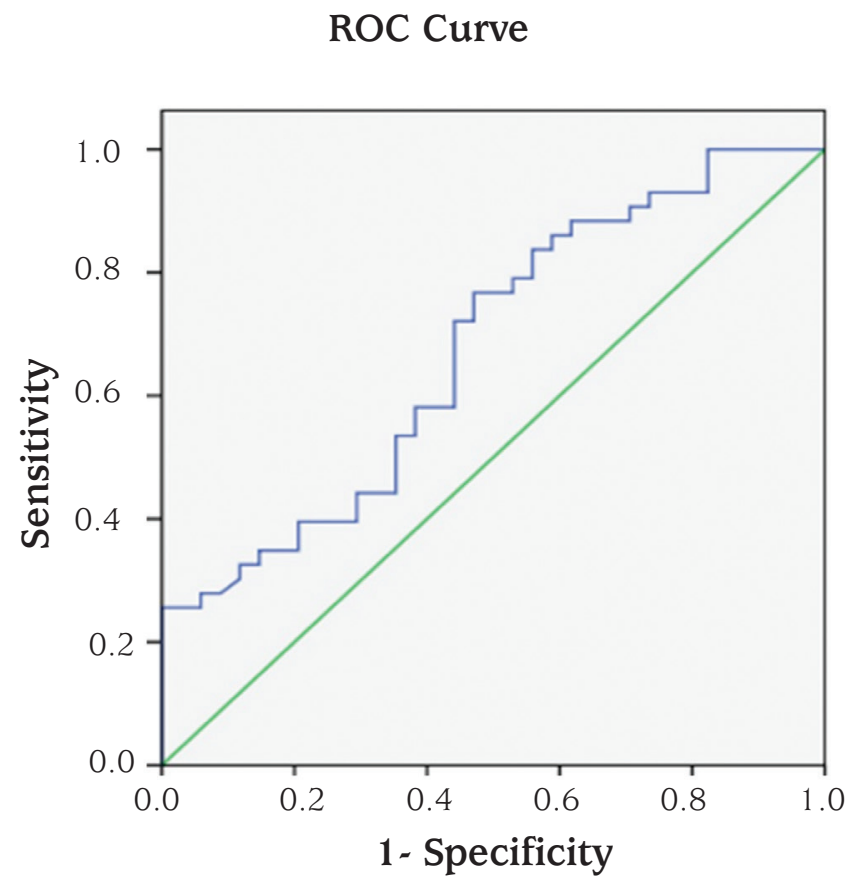

Diagonal segments are produced by ties

Figure 1. Versican receiver operating characteristic curve.

ROC: receiver operating characteristic

The best cut-off value of versican for distinguishing PCOS was 16.49 with $63.64 \%$ sensitivity, $40.73 \%$ specificity, $70.0 \%$ positive and $64.44 \%$ negative predictive values. All statistically significant parameters according to univariate analysis were further evaluated with multivariate logistic regression analysis (Table 2). Considering the laboratory parameters, versican levels less than $33.65 \mathrm{ng} / \mathrm{mL}$ were found to be significantly associated with PCOS (OR: 0.971, 95\% CI: 0.949-0.994, p=0.015). Besides, increased levels of LH were also found to be significantly associated with PCOS (OR: 1.247, $95 \%$ CI: 1.029-1.513, p=0.024).

We used multiple linear regression analysis with stepwise method to evaluate the predictive effects of

\section{ROC Curve}

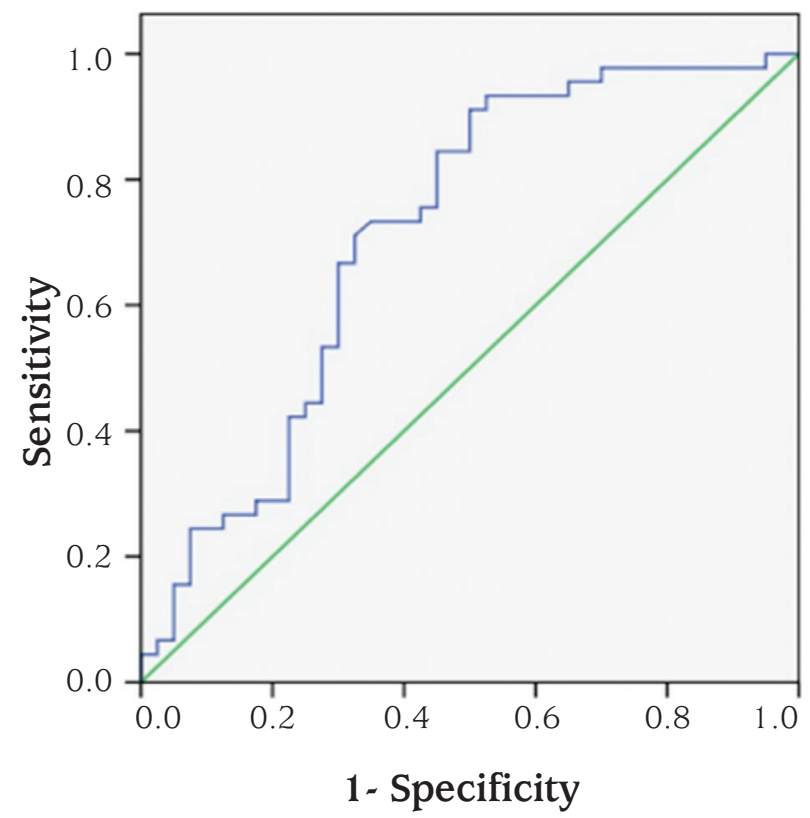

Diagonal segments are produced by ties

Figure 2. Lipid accumulation product index receiver operating characteristic curve.

ROC: receiver operating characteristic

independent variables [like serum versican level, HOMAIR index, Ferriman-Gallwey score (FGS) higher than 8, oligomenorrhea] on PCOS which is a dependent variable. The analysis demonstrated that these parameters were the strongest predictors of PCOS, since they explained $25 \%$ of variance in PCOS (Table 3).

In the PCOS group, a statistically significant positive correlation was determined only between ADAMTS-1 and versican levels $(r=0.615, p<0.001)$. No significant correlations were determined between versican levels and HOMA-IR, LAP index, hyperandrogenism, age at menarche, FGS, and oligomenorrhea.

\section{Discussion}

In the present case-control study, significantly decreased levels of serum versican were detected in adolescents and young adults with PCOS when compared with healthy agematched controls. In addition, a statistically significant positive correlation was determined between serum ADAMTS-1 and versican levels in the PCOS group.

Ovulation requires remodelling of some essential ECM components to permit the release of the COC from the surface of the ovary. This process involves proteolytic 
events, as well as the proper formation of the expanded COC matrix (24). ADAMTS-1 is shown to be secreted mainly by mural granulosa cells, localized to the ECM of expanded COCs and induced markedly by LH in ovulating follicles $(18,25)$. Besides, versican was also shown to be present in the expanded COC matrix and was induced by LH surge, consistent with the activity and localization of ADAMTS-1 $(18,19)$. Xiao et al (26) investigated the expression of ADAMTS-1 in granulosa cells of the PCOS patients, both by immunocytochemistry and reverse transcription polymerase chain reaction and demonstrated the decreased expression of ADAMTS-1 in PCOS patients when compared with normally ovulating women. On the contrary, we found no statistically significant difference in ADAMTS-1 levels between PCOS and the control groups. Although not significant, ADAMTS-1 levels were lower in the PCOS group and, in the present study, we also demonstrated a significant positive correlation between ADAMTS-1 and versican levels. A possible explanation for this discrepancy may be the limited number of participants of our study. However, Russell et al (18) showed that versican was cleaved in the COCs of the progesterone receptor knocked out mice, even when ADAMTS-1 levels were markedly decreased, a finding which is consistent with the results of the present study.

We observed significantly decreased serum versican levels in PCOS patients when compared with the controls, in our study. Consistent with our result, Richards et al (27) demonstrated that versican was the primary substrate in COCs not only for ADAMTS- 1 but also for ADAMTS- 4 and 5 , and they were all expressed in spatiotemporal patterns suggesting evident and probable overlapping functions with each other. So in the present study, as the versican levels were significantly decreased in the PCOS group, we suggested that it was the primary component of the COCs cleaved during ovulation by the induction of LH surge. However, the insignificantly lower levels of ADAMTS- 1 which were observed in our study suggested its important but not the only role in the degradation of ECM components.

LAP index is a cheap and easily available marker of risk for cardiovascular disease. Previous studies have already shown that it has good and reliable diagnostic accuracy for the detection of IR, metabolic syndrome, and risk for cardiovascular disease, even stronger than BMI, WC, and waist-hip ratio $(28,29)$.

We attributed the lack of differences in levels of DHEAS, free- $\mathrm{T}$, total-T, and TG between the groups, to the smallness of our sample. The small number of patients was the main limitation of our study. However, we consider this study important as one of the first in the field and believe that it will encourage further clinical research in this area which include larger samples yielding more significant results.

In conclusion, serum versican levels were found to be significantly decreased in adolescent girls and young women with PCOS. The results of this study support a possible role of versican in ovulatory dysfunction and in the pathogenesis of PCOS.

\section{Ethics}

Ethics Committee Approval: The study protocol was approved by the local ethics committee of our hospital (Approval date/number: 16.10.2014/15). Informed Consent: All participants provided a written informed consent.

Peer-review: Externally peer-reviewed.

\section{Authorship Contributions}

Concept: Sibel Özler, Efser Öztaş, Aytekin Tokmak, Halil İbrahim Yakut, Nafiye Yılmaz, Design: Sibel Özler, Efser Öztaş, Aytekin Tokmak, Halil İbrahim Yakut, Nafiye Yılmaz, Data Collection or Processing: Aytekin Tokmak, Meryem Kuru Pekcan, Başak Gümüş Güler, Analysis or Interpretation: Sibel Özler, Aytekin Tokmak, Literature Search: Sibel Özler, Efser Öztaş, Aytekin Tokmak, Merve Ergin, Meryem Kuru Pekcan, Başak Gümüş Güler, Halil İbrahim Yakut, Nafiye Yılmaz, Writing: Sibel Özler, Efser Öztaş, Merve Ergin, Başak Gümüş Güler, Halil İbrahim Yakut, Nafiye Yılmaz.

Financial Disclosure: The authors declared that this study received no financial support.

\section{References}

1. Ehrmann DA. Polycystic ovary syndrome. N Eng J Med 2005;352:12231236.

2. Lauritsen MP, Bentzen JG, Pinborg A, Loft A, Forman JL, Thuesen LL, Cohen A, Hougaard DM, Nyboe Andersen A. The prevalence of polycystic ovary syndrome in a normal population according to the Rotterdam criteria versus revised criteria including anti-Mullerian hormone. Hum Reprod 2014;29:791-801. Epub 2014 Jan 16

3. Lorenz LB, Wild RA. Polycystic ovarian syndrome: an evidence-based approach to evaluation and management of diabetes and cardiovascular risks for today's clinician. Clin Obstet Gynecol 2007;50:226-243.

4. Rotterdam ESHRE/ASRM-Sponsored PCOS Consensus Workshop Group. Revised 2003 consensus on diagnostic criteria and longterm health risks related to polycystic ovary syndrome. Fertil Steril 2004;81:19-25.

5. Diamanti-Kandarakis E, Christakou C, Marinakis E. Phenotypes and enviromental factors: their influence in PCOS. Curr Pharm Des 2012;18:270-282

6. Rodgers RJ, Irving-Rodgers HF. The roles of the ovarian extracellular matrix in fertility. Soc Reprod Fertil Suppl 2010;67:217-230.

7. Smith MF, Ricke WA, Bakke LJ, Dow MP, Smith GW. Ovarian tissue remodeling: role of matrix metalloproteinases and their inhibitors. Mol Cell Endocrinol 2002;191:45-56 
8. Russell DL, Robker RL. Molecular mechanisms of ovulation: coordination through the cumulus complex. Hum Reprod Update 2007;13:289-312. Epub 2007 Jan 22

9. de Agostini A. An unexpected role for anticoagulant heparan sulfate proteoglycans in reproduction. Swiss Med Wkly 2006;136:583-590.

10. Ny T, Wahlberg P, Brändström IJ. Matrix remodelling in the ovary: regulation and functional role of the plasminogen activator and matrix metalloproteinase systems. Mol Cell Endocrinol 2002;187:29-38.

11. Porter S, Clark IM, Kevorkian L, Edwards DR. The ADAMTS metalloproteinases. Biochem J 2005;386:15-27.

12. Galis ZS, Khatri JJ. Matrix metalloproteinases in vascular remodelling and atherogenesis: the good, the bad, and the ugly. Circ Res 2002;90:251-262.

13. Shozu M, Minami N, Yokoyama H, Inoue M, Kurihara H, Matsushima $\mathrm{K}$, Kuno K. ADAMTS-1 is involved in normal follicular development, ovulatory process and organization of the medullary vascular network in the ovary. J Mol Endocrinol 2005;35:343-355.

14. Zhu H, Leung PC, MacCalman CD. Expression of ADAMTS-5/implantin in human decidual stromal cells: regulatory effects of cytokines. Hum Rep 2007;22:63-74. Epub 2006 Oct 26.

15. Zheng XL. Structure-function and regulation of ADAMTS-13 protease. J Thromb Haemost 2013;11 (Suppl 1):11-23.

16. Robker RL, Russell DL, Espey LL, Lydon JP, O’Malley BW, Richards JS. Progesterone-regulated genes in the ovulation process: ADAMTS- 1 and cathepsin L proteases. Proc Natl Acad Sci USA 2000;97:4689-4694.

17. Espey LL, Yoshioka S, Russell DL, Robker RL, Fujii S, Richards JS. Ovarian expression of a disintegrin and metalloproteinase with thrombospondin motifs during ovulation in the gonadotropin-primed immature rat. Biol Reprod 2000;62:1090-1095.

18. Russell DL, Doyle KM, Ochsner SA, Sandy JD, Richards JS. Processing and localization of ADAMTS- 1 and proteolytic cleavage of versican during cumulus matrix expansion and ovulation. J Biol Chem 2003;278:42330-42339. Epub 2003 Aug 7

19. Russell DL, Ochsner SA, Hsieh M, Mulders S, Richards JS. Hormoneregulated expression and localization of versican in the rodent ovary. Endocrinology 2003;144:1020-1031.
20. Davidsson P, Hulthe J, Fagerberg B, Olsson BM, Hallberg C, Dahllöf B, Camejo G. A proteomic study of the apolipoproteins in LDL subclasses in patients with the metabolic syndrome and type 2 diabetes. J Lipid Res 2005;46:1999-2006. Epub 2005 Jul 1

21. Olsson U, Egnell AC, Lee MR, Lundén GO, Lorentzon M, Salmivirta M, Bondjers G, Camejo G. Changes in matrix proteoglycans induced by insulin and fatty acids in hepatic cells may contribute to dyslipidemia of insulin resistance. Diabetes 2001;50:2126-2132.

22. Matthews DR, Hosker JP, Rudenski AS, Naylor BA, Treacher DF, Turner RC. Homeostasis model assessment: insulin resistance and beta-cell function from fasting plasma glucose and insulin concentrations in man. Diabetologia 1985;28:412-419.

23. Kahn HS. The "lipid accumulation product" performs better than the body mass index for recognizing cardiovascular risk: a populationbased comparison. BMC Cardiovasc Disord 2005:26.

24. Richards JS, Russell DL, Ochsner S, Espey LL. Ovulation: new dimensions and new regulators of the inflammatory-like response. Annu Rev Physiol 2002;64:69-92.

25. Doyle KM, Russell DL, Sriraman V, Richards JS. Coordinate transactivation of the ADAMTS-1 gene by luteinizing hormone and progesterone receptor. Mol Endocrinol 2004;18:2463-2478. Epub 2004 Jul 15

26. Xiao S, Li Y, Li T, Chen M, Xu Y, Wen Y, Zhou C. Evidence for decreased expression of ADAMTS-1 associated with impaired oocyte quality in PCOS patients. J Clin Endocrinol Metab 2014;99:1015-1021. Epub 2014 Mar 19

27. Richards JS, Hernandez-Gonzalez I, Gonzalez-Robayna I, Teuling E, Lo Y, Boerboom D, Falender AE, Doyle KH, LeBaron RG, Thompson V, Sandy JD. Regulated expression of ADAMTS family members in follicles and cumulus oocyte complexes: evidence for specific and redundant patterns during ovulation. Biol Reprod 2005;72:1241-1255. Epub 2005 Jan 19

28. Hosseinpanah F, Barzin M, Erfani H, Serahati S, Ramezani Tehrani F, Azizi F. Lipid accumulation product and insulin resistance in Iranian PCOS prevalence study. Clin Endocrinol (Oxf) 2014;81:52-57. Epub 2013 Aug 9

29. Nascimento JX, Chein MB, de Sousa RM, Ferreira Ados S, Navarro PA, Brito LM. Importance of lipid accumulation product index as a marker of CVD risk in PCOS women. Lipids Health Dis 2015;14:62. 